\title{
26344 - IMMEDIATE POST-PARTUM BLOOD TRANSFUSION : A CHART REVIEW
}

\section{Sudhir Dhumne MD, Shilpa Kasodekar, MD; Michael Moore, FCARCSI; Mrinalini Balki, Jose Carvalho, FRCPC; Mount Sinai Hospital, University Of Toronto, Toronto, ONTARIO, Canada}

Introduction: Obstetric hemorrhage is a major cause of morbidity and mortality. The 2000-02 Confidential Enquiry into Maternal Deaths reported 17 deaths due to hemorrhage, compared to 7 in the previous triennium; 10 were due to postpartum hemorrhage (PPH) compared to one in the previous report.1 Major risk factors for PPH include nulliparity, obesity, macrosomia, multiple pregnancies, prolonged or augmented labors, prolonged third stage, ante-partum hemorrhage, previous $\mathrm{PPH}$ and operative deliveries particularly emergency $\mathrm{C}$-sections (CS). 2 This chart review was undertaken to describe the most common patient characteristics and the clinical management of patients requiring blood transfusions within $24 \mathrm{~h}$ of delivery. Our goal is to use this information to better strategize the clinical care of these patients, including preparedness for blood transfusion.

Methods: After REB approval, a chart review was carried out in all patients receiving blood transfusions in the first $24 \mathrm{~h}$ post-delivery from June 2000 to June 2005.

Results: The blood transfusion rate was $0.31 \%$ (104 patients out of 33,631 deliveries). The causes of PPH were atony $(38.5 \%)$, retained products $(33.7 \%)$ and genital tract trauma (12.5\%). 67 patients (64.4\%) delivered vaginally, 25 (24\%) by emergency CS during labor and only $12(11.5 \%)$ by elective CS. Amongst those delivering vaginally, 61 (58.6\%) were in spontaneous labor and 31(29.8\%) were induced; only $26 \%$ underwent augmentation of labor. As regards duration of labor, 13 (12.5\%) and $6(5.76 \%)$ patients had prolonged 1st and 2nd stages respectively. The majority of patients had normal placentation (82\%). Other important risk factors were multiple gestation (17\%) and PIH (13\%). Lowest values for hemoglobin, hematocrit and platelets were $6.3 \pm 1.5 \mathrm{~g} \%$; $19 \pm 4.4 \% ; 128,000 \pm 74,000 / \mathrm{mm} 3$. The blood products (mean) transfused were as follows: PRBC (4.9 units) in 100\% patients; FFP (5.9 u) in $42 \%$ patients; platelets (7.4 u) in 18\% patients; cryoprecipitate $(6 \mathrm{u})$ in $9 \%$ patients. The uterotonic agents (mean) used were as follows: oxytocin (47 iu) in $100 \%$ patients; ergot $(0.45 \mathrm{mg})$ in $56 \%$ patients; carboprost $(0.73 \mathrm{mg})$ in $49 \%$ patients; misoprostol $(976 \mathrm{mg})$ in $24 \%$ patients. Dilatation and curettage (26.9\%), manual removal of placenta (17.3\%), hysterectomy (16.3\%) and uterine artery embolization $(16.3 \%)$ were the most common surgical procedures performed. $21 \%$ of patients developed coagulopathy and $24 \%$ required admission to ICU. No deaths occurred.

Discussion: Severe PPH requiring blood transfusion is unpredictable. Most cases are healthy laboring patients with no identified risk factors. A better prophylactic pharmacological strategy and early recognition of retained products might reduce the incidence of blood transfusion, coagulopathy and ICU admission. We suggest that all laboring patients should undergo blood group typing and screening.

References:

1. Why Mothers Die 2000-2002 http://www.cemach.org.uk/

2. Obstet Gynecol 1991; 77:77-82 